\title{
Connectivity Graphs for Single Zigzag Chains and their Application for Computing ZZ Polynomials
}

\author{
Johanna Langner, Henryk A. Witek*
}

\author{
Department of Applied Chemistry and Institute of Molecular Science, National Chiao Tung University, Hsinchu, Taiwan \\ * Corresponding author's e-mail address: hwitek@mail.nctu.edu.tw
}

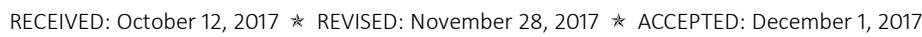

PROCEEDING OF THE 29 ${ }^{\text {TH }}$ MATH/CHEM/COMP CONFERENCE, JUNE 19-25, 2017, DUBROVNIK, CROATIA THIS PAPER IS DEDICATED TO PROFESSOR IVAN GUTMAN ON THE OCCASION OF HIS 70 ${ }^{\mathrm{TH}}$ BIRTHDAY

\begin{abstract}
We present a graph-theoretical interpretation of the recently developed interface theory of single zigzag chains $N(n)$ in form of connectivity graphs. A remarkable property of the connectivity graphs is the possibility of constructing the full set of Clar covers of $N(n)$ as the complete set of walks on these graphs. Connectivity graphs can be constructed in a direct form, in which the number of vertices is growing linearly with the length $n$ of $N(n)$, and in a reduced form, in which the number of vertices is independent of the actual length of the chain. The presented results can be immediately used for the determination of the Zhang-Zhang (ZZ) polynomials of $N(n)$ in an easy and natural manner. Two techniques for computing the ZZ polynomial are proposed, one based on direct recursive computations and the other based on a general solution to a set of recurrence relations. Generalization of the interface theory to arbitrary benzenoid structures, the construction of associated connectivity graphs, and techniques for the computation of the associated ZZ polynomials will be presented in the near future.
\end{abstract}

Keywords: benzenoids, Clar cover, interface theory, connectivity graph, Zhang-Zhang polynomial, ZZ polynomial.

\section{INTRODUCTION}

A LGORITHMS and techniques for generating resonance structures of benzenoids always attracted the attention of the chemical community. In a usual approach, one generates the full set of resonance structures via recursive decomposition of a given benzenoid $S$, where the term decomposition refers to assigning definitive character to each chemical bond in S. ${ }^{[1-4]}$ The decomposition process can be illustrated in a form of a tree-like graph, in which each node corresponds to the assignment of a definitive character to a consecutive bond in the bond list of $S$. The complete set of resonance structures is isomorphic to the collection of leaves of the resulting tree-like graph. The Clar covers (i.e., generalized resonance structures) of benzenoid hydrocarbons constructed this way can be conveniently enumerated using ZZ polynomials (aka Zhang-Zhang polynomials or Clar covering polynomials). Already for relatively small benzenoid structures, the number of conceivable resonance structures and consequently the size of the tree-like decomposition graph are humongous, preventing one from visualizing, studying, and analyzing the decomposition graph in its entire form. This problem seems to be unavoidable since the number of vertices in any decomposition graph needs to be at least as large as the number of conceivable resonance structures. An example of such a decomposition tree generating the complete set of Clar covers of a relatively small benzenoid, the single zigzag chain $N(n)$ with $n=5$, is shown in Figure 1.

The theory of Clar covers and associated ZZ polynomials ${ }^{[4-13]}$ constitutes a dynamic field of research in chemical graph theory. Over the past few years our group has been actively involved in this research. In particular, we have developed a computer code called ZZCalculator, [3] which is capable of computing ZZ polynomials of small and medium-size benzenoids, and a collection of analytical methods available as ZZDecomposer, ${ }^{[4]}$ which can be used for discovering recurrence relations between isostructural benzenoids. Such recurrence relations allow derivation of closed-form ZZ polynomials for whole classes of benzenoid structures at once, ${ }^{[8,14,15]}$ as has been demonstrated with the help of ZZDecomposer for many classes of 


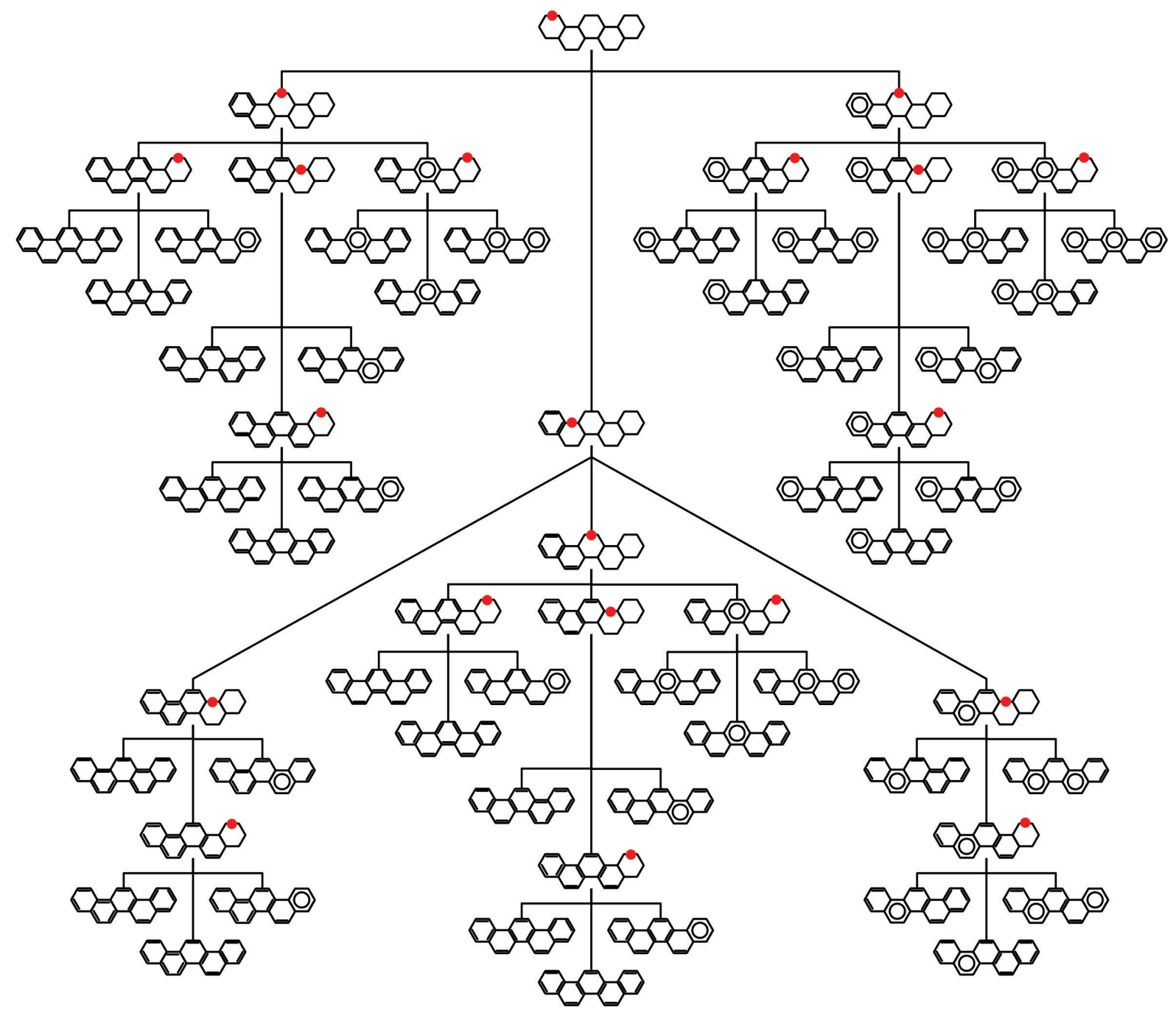

Figure 1. Decomposition trees used for constructing Clar Covers of benzenoid structures can be enormously large as illustrated here for the single zigzag chain $N(5)$. Structures with dots indicate intermediate stages.

benzenoids. ${ }^{[4,11,13,16-19]}$ These approaches are very useful in many situations, but when the size of the structure $S$ is too large or when there is no apparent structure allowing to relate the intermediate structures in the decomposition process, ZZDecomposer and ZZCalculator are not able to produce the $\mathrm{ZZ}$ polynomial of $S$ due to the prohibitive computational complexity of the underlying decomposition process.

The ideas presented in the current manuscript, based on the interface theory, are hoped to lift these limitations. We propose a novel way of decoding the complete set of the resonance structures using certain directed graphs, introduced here under the name of connectivity graphs. These graphs are constructed here for a single class of benzenoid structures (single zigzag chains $N(n)$ ), but the generalization to an arbitrary class of benzenoids is rather straightforward. We show explicitly that the resonance structures of $N(n)$ can be represented as walks on the connectivity graphs. This property holds also for other classes of benzenoids. Representing resonance structures as paths on connectivity graphs allows one to keep the number of vertices in connectivity graphs small and results in a dense and compact structure of these objects in contrast to the decomposition trees. Furthermore, connectivity graphs can be utilized in a straightforward way to compute the ZZ polynomials for single zigzag chains $N(n)$. These remarkable properties of connectivity graphs are an immediate consequence of the recently introduced interface theory of benzenoid structures. The first part of the current paper gives a short overview of the Clar cover theory and the interface theory as applicable to single zigzag chains $N(n)$. The second part demonstrates how some of the presented theorems concerning the connectivity patterns between fragments 
and interfaces can be represented graphically in the form of connectivity graphs. In this sense, the connectivity graphs are an inherent part of the interface theory and can be constructed for an arbitrary class of benzenoids. An interesting alternative form of the connectivity graphs are the reduced connectivity graphs, which do not depend on the length of the benzenoid and correspond simultaneously to an entire class of structures. In the third part of this paper, we show how, for an arbitrary value of $n$, the total number of Clar covers and the ZZ polynomial ZZ $(N(n), x)$ can be extracted from the interface connectivity graph. The actual computation process proceeds via the recurrence relations that are extracted from the interface connectivity graph. A standard solution to these recurrence equations produces the total number of Clar covers and the $\mathrm{ZZ}$ polynomial of single zigzag chains for an arbitrary $n$. Interestingly, the ZZ polynomial of $N(n)$ can be transformed to a new structural form, which explicitly reflects the number of Clar covers of each order in a short and compact expression. Generalization of the ideas presented in the current paper for single zigzag chains to an arbitrary class of benzenoid structures will be presented shortly.

\section{PRELIMINARIES}

A benzenoid hydrocarbon $S$ is a condensed polycyclic aromatic compound made up entirely out of fused benzene rings. ${ }^{[20]} \mathrm{A}$ Clar cover of $S$ can be defined ${ }^{[21]}$ as a generalized resonance structure of $S$, such that every carbon atom maintains tetravalent character by participating in either a double bond or an aromatic sextet. The order of a given Clar cover $C$ is defined as the number of aromatic rings present in $C$. The number of conceivable Clar covers of $S$ can be conveniently expressed in a form of a Clar covering polynomial (aka Zhang-Zhang polynomial or ZZ polynomial) $[1,22,24]$

$$
\mathrm{ZZ}(S, x)=\sum_{k=0}^{c} c_{k} x^{k},
$$

where the Clar number $\mathrm{Cl}$ is the maximal number of aromatic rings that can appear in $S^{[21,25]}$ and $c_{k}$ is the number of possible Clar covers of order $k$. To illustrate these concepts, we show in Figure 2 all Clar covers of a single zigzag chain ${ }^{[26]}$ of length four (abbreviated as $N(4)$ ), grouped by their respective order, together with the resulting ZZ polynomial of $N(4)$.

The computation of $\mathrm{ZZ}$ polynomials is a straightforward process owing to the existing recursive decomposition algorithms. ${ }^{[2-4]}$

A single zigzag chain $N(n)$ of length $n$ can be defined as a sequence of $n$ cata-condensed benzene rings selected from the hexagonal lattice in the armchair direction, as shown in Figure 3. ${ }^{[3,26]}$ Single zigzag chains are sometimes

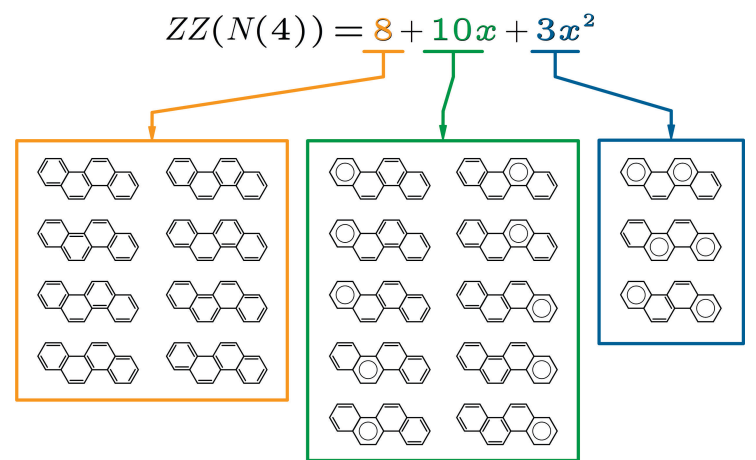

Figure 2. The set of Clar covers of a zigzag chain N(4) and the associated ZZ polynomial.

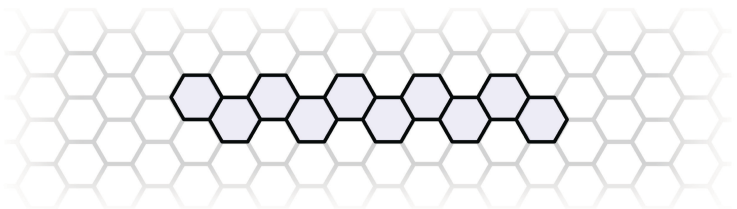

Figure 3. A single zigzag chain $N(n)$ of length $n$ consists of $n$ cata-condensed benzene rings selected from the hexagonal lattice in the armchair direction, as illustrated here for $n=10$.

referred to as single armchair chains ${ }^{[3]}$ or just as zigzag chains.

\section{INTERFACE THEORY}

This section gives a short introduction into the interface theory and summarizes the essential definitions and results. For a detailed introduction, see Ref. [27]. First, we define two objects that can serve as descriptors of the local structure of a Clar cover: interfaces and fragments. Both can be used to construct unique sequence representations of Clar covers. The representation of Clar covers using fragments is intuitively more obvious and will be introduced first. However, the representation of Clar covers using interfaces appears to be more compact, convenient and promising for practical application.

\section{Fragment Formulation}

Assume a single zigzag chain $N(n)$ is horizontally oriented as depicted in Figure3. Consider vertical partition lines cutting through the center of each hexagon as depicted in Figure 4. Between any two adjacent partition lines lies a segment of $N(n)$, which consists of one or two fused halfhexagons. The full set of segments $\{a, b, c, d, e, f\}$ that can be obtained by partitioning a single zigzag chain is shown in Figure 4. A sequence of segments $X=\left(x_{1}, x_{2}, \ldots, x_{n+1}\right)$ that represents some zigzag chain $N(n)$ will be called a generating sequence. It is easy to see that $N(n)$ is uniquely represented by the generating sequence $(a, d, c, d, \ldots, c, d, c, e)$ 


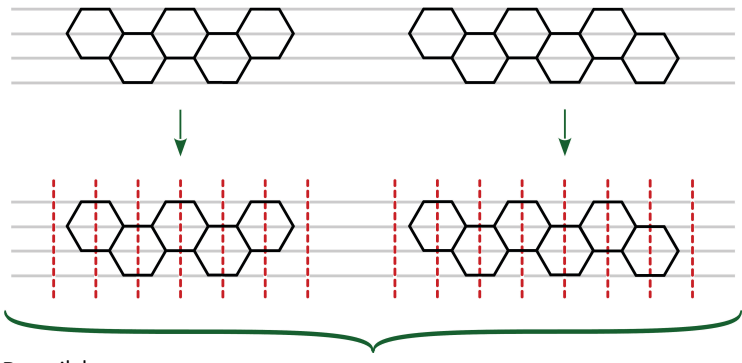

Possible segments:

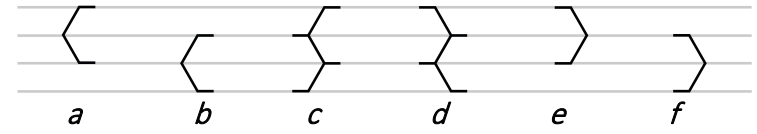

Figure 4. Partitioning of a single zigzag chain produces six possible segments $(a-f)$.

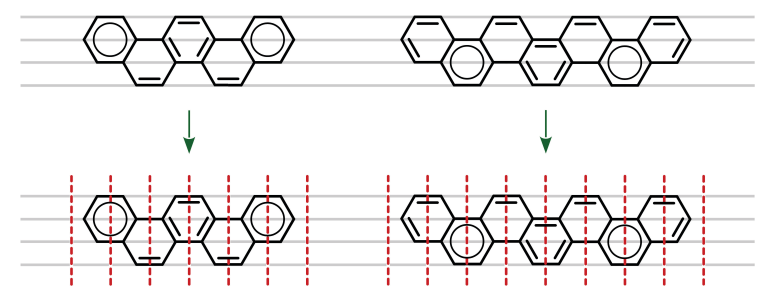

Figure 5. Examples of partitioning Clar covers of single zigzag chains into fragments.
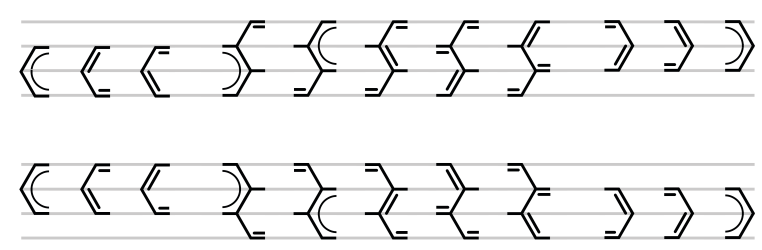

Figure 6. Full set of fragments for a single zigzag chain.

if $n$ is odd, and by the generating sequence $(a, d, c, d, \ldots$, $c, d, c, d, f)$ if $n$ is even. ${ }^{[27-C o r .2] ~}$

Clar covers of the segments $a-f$ are referred to as fragments. The full set of fragments that can be obtained by partitioning a Clar cover of a single zigzag chain, as shown in Figure 5, is given by Figure 6.[27-Lem.5,19]

For each Clar cover of $N(n)$, the partitioning process illustrated in Figure 5 produces a sequence of fragments. A sequence of fragments $F=\left(f_{1}, f_{2}, \ldots, f_{n+1}\right)$ will be referred to as a covering sequence if there is a Clar cover $C$ that can be partitioned into $F$. There exists a one-to-one correspondence between the set of Clar covers and the set of covering sequences.[27-Thm.13] In other words, every Clar cover $C$ of $N(n)$ is uniquely represented by a covering sequence $F=\left(f_{1}, f_{2}, \ldots, f_{n+1}\right)$.
However, not every sequence of fragments generates a Clar cover. Roughly speaking, in a covering sequence neighboring elements must fit together with respect to both their geometric shapes and their Clar coverings. The precise condition for whether a sequence of fragments is a covering sequence or not can be formulated after the concept of an interface is introduced in the next subsection.

\section{Interface Formulation}

Consider a single zigzag chain $N(n)$ that is horizontally oriented as depicted in Figure 3. Each hexagon $h_{j}$ in $N(n)$ possesses a pair of horizontal edges. Specifically, we can distinguish an outer edge, which connects vertices that belong only to the hexagon $h_{j}$, and an inner edge, which has at least one vertex belonging to an adjacent hexagon $h_{j-1}$ or $h_{j+1}$. The Clar covering character of this pair of horizontal edges, denoted as $(k l)$ will be referred to as an interface. In the symbol $(k l), k$ refers to the outer edge and $/$ refers to the inner edge. The indices $k$ and $/$ can take the values $s, d$ and a, corresponding to single, double and aromatic bonds, respectively. In graph-theoretical terminology, the values s, $\mathrm{d}$ and a correspond to no covering, edge covering $\left(K_{2}\right)$ and hexagon covering $\left(C_{6}\right)$, respectively. Each fragment as defined above possesses a left and/or a right interface, as shown in Figure 7.

Every Clar cover of a single zigzag chain can be expressed a sequence of interfaces. A sequence of interfaces $I=\left(i_{1}, i_{2}, \ldots, i_{n}\right)$ will be referred to as a linking sequence if there exists a Clar cover $C$ of $N(n)$ such that $i_{j}$ is the interface of the $j^{\text {th }}$ hexagon of $N(n)$. Alternatively and equivalently, we can say that a sequence of interfaces $I=\left(i_{1}, i_{2}, \ldots, i_{n}\right)$ is a linking sequence if there exists a covering sequence $F=\left(f_{1}, f_{2}, \ldots, f_{n+1}\right)$ such that $i_{j}$ is the right interface of the fragment $f_{j}$ for $1 \leq j \leq n$. There exists a one-to-one correspondence between the set of Clar covers and the set of linking sequences. ${ }^{[27-C o r .17]}$ In other words, every Clar cover $C$ of $N(n)$ is uniquely represented by a linking sequence $I=\left(i_{1}, i_{2}, \ldots, i_{n}\right)$.

However, not every sequence of interfaces is a linking sequence. Below, we give the necessary and sufficient conditions both for covering sequences and linking sequences.

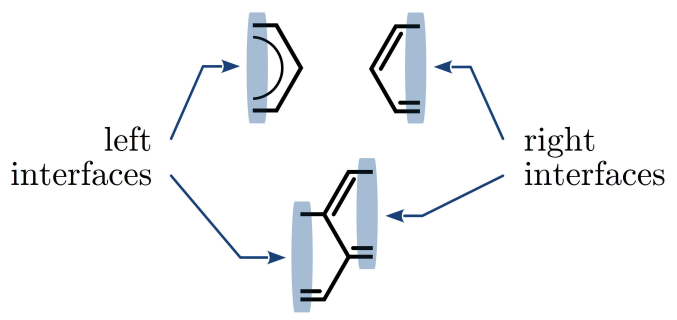

Figure 7. Interfaces of fragments. 

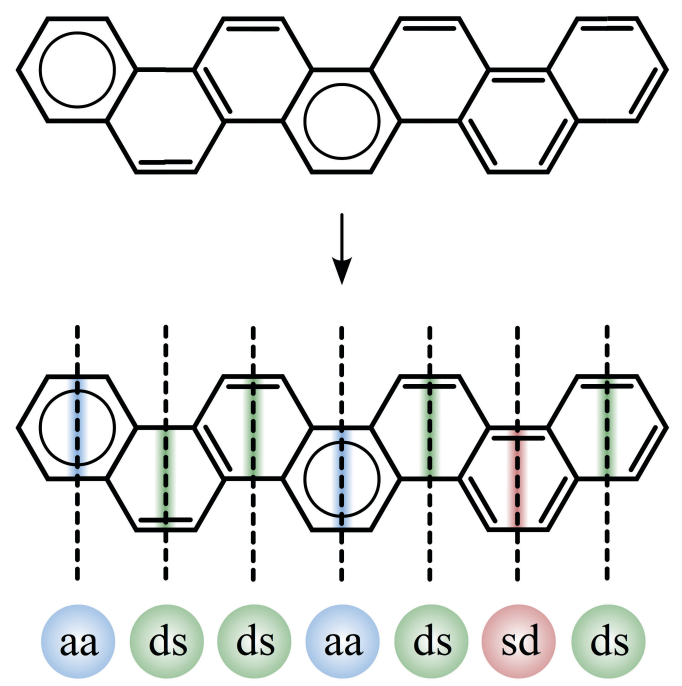

Figure 8. Construction of a linking sequence from a Clar cover. Every vertical line cutting through the center of a hexagonal face crosses a pair of horizontal edges. The Clar covering character of the outer and inner edges ( $k$ and $l$, respectively) determines the interface symbol $(k l)$. The possible interfaces are: $(\mathrm{aa})=\mathrm{aa},(\mathrm{sd})=\mathrm{sd},(\mathrm{ds})=\mathrm{ds}$.

Theorem 1. Ref. [27-Thm.12] A sequence of fragments $F=\left(f_{1}, \ldots, f_{n+1}\right)$ is a covering sequence if and only if

- The underlying sequence of segments $\left(x_{1}, x_{2}, \ldots, x_{n+1}\right)$ is a generating sequence, where the segments $x_{i}$ are obtained by removing the covering from $f_{i}$.

- The right interface of the fragment $f_{j}$ is identical to the left interface of the fragment $f_{i+1}$ for all $1 \leq j \leq n$.

Theorem 2. Ref. [27-Thm.21] A sequence of interfaces $I=\left(i_{1}, \ldots, i_{n}\right)$ is a linking sequence if and only if

- $i_{j} \in\{(\mathrm{ds}),(\mathrm{sd}),(\mathrm{aa})\} \forall 1 \leq j \leq n$,

- every pair $\left\{i_{j}, i_{j+1}\right\}$ of consecutive interfaces contains at least one (ds) interface, (ds) $\in\left\{i_{j}, i_{j+1}\right\} \forall 1 \leq j \leq n-1$.

The conditions in Theorems 1 and 2 enable us to construct the full sets of such sequences. In Ref. [27], this was achieved by an recursive algorithm. Here, we solve this problem in a different way, namely by constructing certain directed graphs that incorporate the conditions given by Theorem 2 and, as a result, encode the complete set of Clar covers of $N(n)$. These graphs will be referred to as connectivity graphs.

\section{CONNECTIVITY GRAPHS}

In this section, we introduce connectivity graphs for benzenoid structures. In principle, such graphs could be constructed in several different ways, the main distinction being the choice of vertices. Here we restrict our attention to the most useful possibility, the interface connectivity graphs, in which the vertices correspond to interfaces, and the edges correspond to fragments. These graphs can be used to construct the full set of linking sequences, and consequently the full set of Clar covers of the benzenoid at hand. Detailed construction of interface connectivity graphs of a single zigzag chain $N(n)$ is presented in the sections below.

\section{Interface Connectivity Graphs}

To construct an interface connectivity graph for a single zigzag chain $N(n)$, we start by taking its generating sequence $X=\left(x_{1}, x_{2}, \ldots, x_{n+1}\right)$, constructing all possible Clar covers $\left\{f_{j}^{k}\right\}$ of the segments $x_{j}$, and taking the set of all their right interfaces. Since there are several fragments with identical left or right interfaces, the set $\left\{i_{j}^{l}\right\}$ of their conceivable right interfaces is much smaller than the set $\left\{f_{j}^{k}\right\}$ of fragments. We know from Theorem 2 that $\left\{i_{j}^{\prime}\right\}=\left\{(\mathrm{ds})_{\mathrm{j}},(\mathrm{sd})_{\mathrm{j}},(\mathrm{aa})_{\mathrm{j}}\right\}$ for every $j=1, \ldots, n$. The set $\left\{i_{j}^{\prime}\right\}$ together with two additional vertices designated as start and end constitute the complete set of vertices of the interface connectivity graph. The complete set of directed edges consists of three distinct classes:

- start $\rightarrow i_{1}^{l}$, which exist if and only if there is a terminal fragment $f_{1}^{k}$ having $i_{1}^{l}$ as the right interface,

- $i_{j}^{l} \rightarrow i_{j+1}^{m}$, which exist if and only if there is a fragment $f_{j}^{k}$ having $i_{j}^{l}$ as the left interface and $i_{j+1}^{m}$ as the right interface,

- $i_{n}^{l} \rightarrow$ end, which exist if and only if there is a terminal fragment $f_{n+1}^{k}$ having $i_{n}^{\prime}$ as the left interface.

Using Theorem 2, we can reformulate the set of directed edges without explicitly mentioning the fragments:

- start $\rightarrow i_{1}^{\prime}$,

- $i_{j}^{l} \rightarrow i_{j+1}^{m}$, which exist if and only if $i_{j}^{l}=(\mathrm{ds})_{j}$ or $i_{j+1}^{m}=(\mathrm{ds})_{j+1}$,

- $i_{n}^{l} \rightarrow$ end

An example of such an interface connectivity graph, constructed for the zigzag chain $N(5)$, is depicted in Figure 9. Note that every edge in Figure 9 represents a unique fragment.

Let us now consider a walk originating at the vertex start, passing through the interfaces $i_{1}^{l_{1}}, i_{2}^{l_{2}}, \ldots, i_{n}^{l_{n}}$, and terminating at the vertex end. Then, according to Theorem 2 , the sequence $\left(i_{1}^{l_{1}}, i_{2}^{l_{2}}, \ldots, i_{n}^{l_{n}}\right)$ is a linking sequence representing some Clar cover of $N(n)$. The order of this Clar cover is given by the number of double-line arrows $\Longrightarrow$ (leading to the interface (aa)) traversed during this walk. 


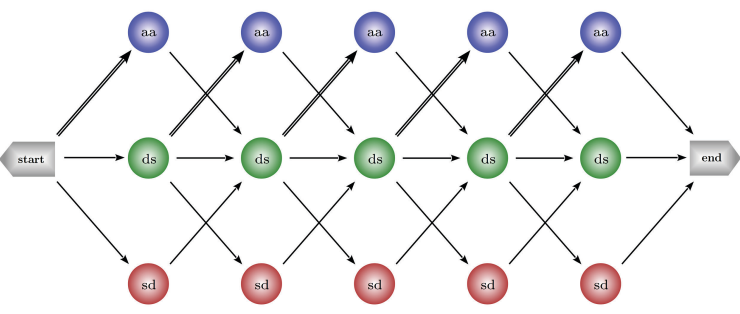

Figure 9. Interface connectivity graph for $N(5)$.

Conversely, every Clar cover is uniquely represented by a linking sequence, which in turn corresponds to a unique walk on the interface connectivity graph. It follows that the complete set of walks from start to end on the interface connectivity graph is isomorphic to the set of Clar covers of $N(n)$.

\section{Kekulé Structures}

In the interface connectivity graph, Kekule structures are represented by walks from start to end that do not pass through any (aa) interface vertices. It follows that the complete set of Kekulé structures of $N(n)$ can be constructed from the full set of such walks.

Note that the interface connectivity graph does not contain any $(\mathrm{sd})_{j} \rightarrow(\mathrm{sd})_{j+1}$ edges. This observation is closely related to the fact that Fibonacci cubes are the resonance graphs of single zigzag chains as well as other fibonaccenes. ${ }^{[28]}$ The vertices of a Fibonacci cube are Fibonacci strings, i.e., binary strings $\left(b_{1} b_{2} \ldots b_{n}\right)$ such that every pair $\left\{b_{j}, b_{j+1}\right\}$ of consecutive digits contains at least one 0 . In the interface theory, every Kekulé structure of a single zigzag chain is represented by a walk from start to end on the interface connectivity graph that does not pass through any (aa) interfaces, i.e., a linking sequence $\left(i_{1}, i_{2}, \ldots, i_{n}\right)$ with $i_{j} \in\{(\mathrm{sd}),(\mathrm{ds})\}$ such that every pair of consecutive interfaces $\left\{i_{j}, i_{j+1}\right\}$ contains at least one (ds) interface. Thus, we see directly that such a walk on the interface connectivity graph - by interpreting every (ds) as a 0 and every (sd) as a - can be seen as a Fibonacci string, i.e., a vertex of a Fibonacci cube. In a resonance graph, two vertices are connected by an edge if the symmetric difference of their corresponding Kekulé structures is exactly one hexagon. In the language of the interface theory for single zigzag chains, this is the case if the linking sequences of the two Kekule structures differ in exactly one interface. Then, the corresponding Fibonacci strings differ in exactly one digit, meaning that, as vertices of a Fibonacci cube, they are connected by an edge. This constitutes a compact alternate proof that Fibonacci cubes are the resonance graphs of single zigzag chains to the one given in Ref. [28].

\section{Reduced Interface Connectivity Graphs}

A certain disadvantage of the interface connectivity graph introduced in the previous section is the fact that every single zigzag chain $N(n)$ requires a different graph. It would be convenient to construct connectivity graphs that would be independent on the length of the chain. This is possible by introducing a reduced set of vertices, employing bidirectional edges and allowing cycles. The resulting graph - referred to as the reduced interface connectivity graph - is depicted in Figure 10. This graph consists of five vertices (the start point, the end point, and the three interfaces that can appear in single zigzag chains, (aa), (ds), and (sd)) and nine oriented edges belonging to three distinct classes:

- start $\rightarrow i^{l}$, which exist if and only if there is a terminal fragment with $i$ as the right interface,

- $i^{l} \rightarrow i^{m}$, which exist if and only if there is an interior fragment with $i^{l}$ as the left interface and $i^{m}$ as the right interface,

- $i^{\prime} \rightarrow$ end, which exist if and only if there is a terminal fragment with $i^{l}$ as the left interface.

Using Theorem 2, we can reformulate the set of directed edges without explicitly mentioning the fragments:

$$
\begin{aligned}
& \text { - start } \rightarrow i^{\prime}, i^{\prime} \in\{(\mathrm{ds}),(\mathrm{sd}),(\mathrm{aa})\}, \\
& \text { - } i^{\prime} \rightarrow i^{m} \text {, which exist if and only if (ds) } \in\left\{i^{l}, i^{m}\right\}, \\
& \text { - } i^{\prime} \rightarrow \text { end. }
\end{aligned}
$$

The complete set of Clar covers of a zigzag chain $N(n)$ is then

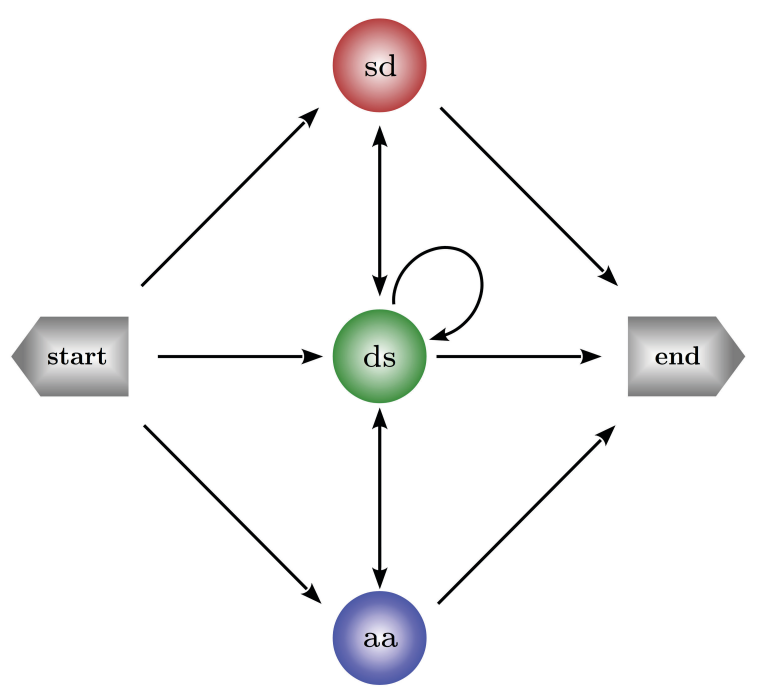

Figure 10. Reduced interface connectivity graph for single zigzag chains. 
isomorphic to the complete set of walks of length $n+1$ on the reduced interface connectivity graph that begin at the vertex start and finish at the vertex end. ${ }^{[27-T h m .22]}$ The structure of the reduced interface connectivity graph is strikingly simple owing to the small number of distinct interfaces, which is much smaller than the number of distinct fragments.

\section{AN ALTERNATIVE WAY OF COMPUTING ZZ POLYNOMIALS FOR SINGLE ZIGZAG CHAINS}

For a given single zigzag chain $N(n)$, all possible linking sequences - and thus, all possible Clar covers of $N(n)-$ are encoded in its corresponding interface connectivity graph. Therefore, it is natural to expect that the ZhangZhang polynomial ZZ $(N(n), x)$, which enumerates those Clar covers, is also encoded in some way in the interface connectivity graph of $N(n)$. To find a way of extracting $\mathrm{ZZ}(N(n), x)$ from this graph, let us first extract the total number of Clar covers $D$, given by

$$
D=\sum_{k=1}^{C l} c_{k}=Z Z(N(n), 1),
$$

which is obviously equal to the number of distinct ways of traversing the interface connectivity graph from $i_{0} \equiv$ start to $i_{n+1} \equiv$ end . To compute it, we introduce the weight $w\left(i_{j}\right)$ of each vertex $i_{j}$ in the interface connectivity graph, which is equal to the number of distinct paths from start to $i_{j}$. We obviously have $w\left(i_{0}\right)=w($ start $)=1$. The weight $w\left(i_{j+1}\right)$ is given recursively by

$$
w\left(i_{j+1}\right)=w\left(i_{j}^{1}\right)+\ldots+w\left(i_{j}^{s}\right),
$$

where $\left\{i_{j}^{1}, \ldots, i_{j}^{s}\right\}$ is the complete set of direct predecessors of $i_{j+1}$. Then, in particular, we have

$$
\begin{aligned}
& w\left((\mathrm{ds})_{j+1}\right)=w\left((\mathrm{ds})_{j}\right)+w\left((\mathrm{sd})_{j}\right)+w\left(\left(\mathrm{aa}_{j}\right),\right. \\
& w\left((\mathrm{sd})_{j+1}\right)=w\left((\mathrm{ds})_{j}\right) \\
& w\left((\mathrm{aa})_{j+1}\right)=w\left((\mathrm{ds})_{j}\right)
\end{aligned}
$$

which can be folded to

$$
w\left((\mathrm{ds})_{j+1}\right)=w\left((\mathrm{ds})_{j}\right)+2 w\left((\mathrm{ds})_{j-1}\right)
$$

with the initialization $w\left((\mathrm{ds})_{0}\right) \equiv w($ start $)=1$ and $w\left((\mathrm{ds})_{1}\right)=1$. Solution to this recurrence relation with constant coefficients is obvious and given by

$$
w\left((\mathrm{ds})_{j}\right)=\frac{1}{3}\left(2^{j+1}-(-1)^{j+1}\right) .
$$

Then the total number of Clar covers $D$ is given by

$$
\begin{aligned}
D & =w(\text { end }) \\
& =w\left((\mathrm{ds})_{n}\right)+w\left((\mathrm{sd})_{n}\right)+w\left((\mathrm{aa})_{n}\right) \\
& =w\left((\mathrm{ds})_{n}\right)+2 w\left((\mathrm{ds})_{n-1}\right) \equiv w\left((\mathrm{ds})_{n+1}\right) \\
& =\frac{1}{3}\left(2^{n+2}-(-1)^{n+2}\right) .
\end{aligned}
$$

The Zhang-Zhang polynomial $Z Z(N(n), x)$ can be computed in exactly the same way after the weight $w\left(i_{j}\right)$ of each vertex $i_{j}$ is replaced with a more general concept, the polynomial weight $W\left(i_{j}\right)$. To introduce $W\left(i_{j}\right)$, we first define the order $o(p)$ of a walk $p$ as the number of the (aa) vertices in $p$. Then the polynomial weight $W\left(i_{j}\right)$ of the vertex $i_{j}$ is defined as

$$
W\left(i_{j}\right)=\sum_{k=0}^{j} c_{k}\left(i_{j}\right) x^{k} \equiv \sum_{p \in P\left(i_{j}\right)} x^{o(p)},
$$

where $c_{k}\left(i_{j}\right)$ represents the number of distinct walks of order $k$ from start to $i_{j}$, and $P\left(i_{j}\right)$ is the set of walks from start to $i_{j}$. In a close analogy to $w\left(i_{j}\right)$, the polynomial weight $W\left(i_{j}\right)$ enumerates the walks from start to $i_{j}$, but does it in a more elaborate way, counting walks of different order separately. Since every walk from start to end of order $k$ represents a Clar cover of the same order $k$, it is clear that $W($ end $)=Z Z(N(n), x)$.

Practical computation of the polynomial weights $W\left(i_{j}\right)$ can be carried out using recursive techniques. We obviously have $W\left(i_{0}\right) \equiv W($ start $)=1$ and $W\left(i_{n+1}\right) \equiv$ $W($ end $)=Z Z(N(n), x)$. The polynomial weight $W\left(i_{j+1}\right)$ of the vertex $i_{j+1}$ can be expressed using the following recurrence relation:

$$
W\left(i_{j+1}\right)=\left\{\begin{array}{l}
x \cdot \sum_{z \in Z} W(z) \quad \text { if } i_{j+1}=(\mathrm{aa})_{j+1}, \\
1 \cdot \sum_{z \in Z} W(z) \quad \text { otherwise }
\end{array}\right.
$$

where $Z$ is the set of direct predecessors of the vertex $i_{j+1}$. This can be easily seen by decomposing each walk $p \in P\left(i_{j+1}\right)$ into a sole vertex $i_{j+1}$ and a walk $q$ one step shorter, which finishes at some direct predecessor $z$ of $i_{j+1}$. Then, the order of $p$ is given by the order of $q$ plus the order of the vertex $i_{j+1}$, which is equal to 1 if $i_{j+1}=(\mathrm{aa})_{j+1}$ and to 0 otherwise. This consideration translates into the following train of equalities

$$
\begin{aligned}
W\left(i_{j+1}\right) & =\sum_{p \in P\left(i_{j+1}\right)} x^{o(p)} \\
& =\sum_{z \in Z} \sum_{q \in P(z)} x^{o(q)+o\left(i_{j+1}\right)} \\
& =x^{o\left(i_{j+1}\right)} \sum_{z \in Z} \sum_{q \in P(z)} x^{o(q)} \\
& =x^{o\left(i_{j+1}\right)} \sum_{z \in Z} W(z),
\end{aligned}
$$

which proves Equation (9). The recursive algorithm based on Equation (9) provides a straightforward way of 


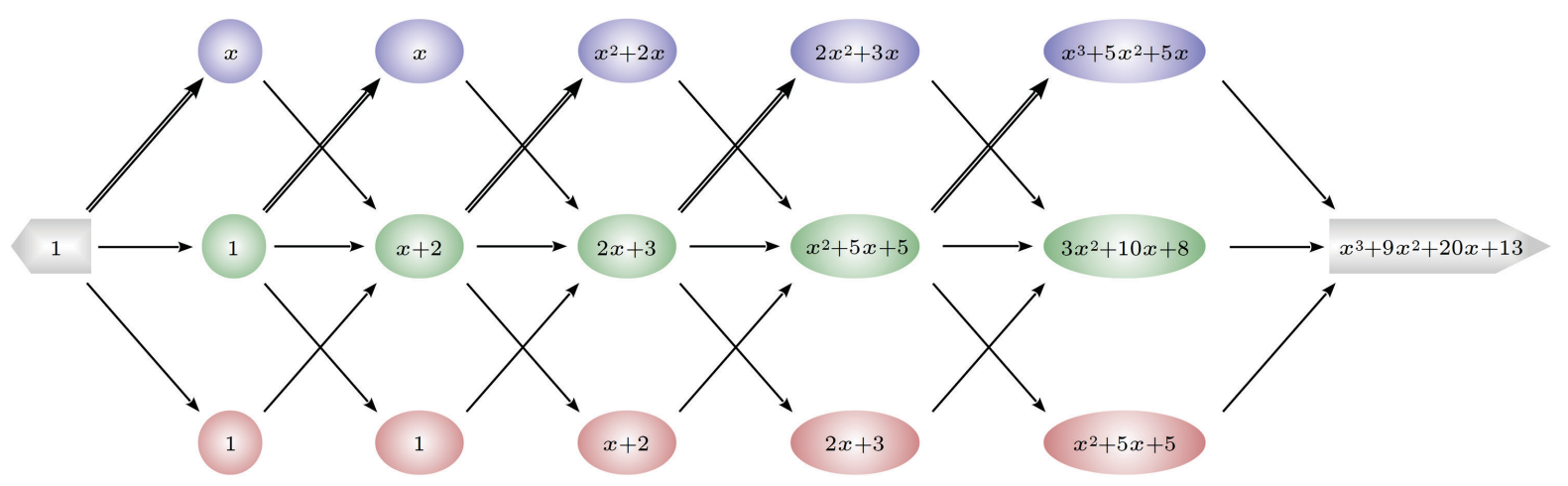

Figure 11. Determination of the ZZ polynomial for a single zigzag chain $N(n)$ using the interface connectivity graph can be readily performed using simple mnemonic rules: $i$ ) the arrow $\longrightarrow$ propagates the polynomial weight of the previous vertex to the next vertex, ii) the arrow $\Longrightarrow$ multiplies the polynomial weight of the previous vertex by $x$ and propagates it to the next vertex, iii) the contributions from all incoming arrows are summed. The ZZ polynomial of $N(n)$ is obtained as the polynomial weight of the end vertex. Note that the ZZ polynomials of all shorter single zigzag chains appear as polynomial weights of the consecutive (ds) interfaces in the central row.

constructing the $\mathrm{ZZ}$ polynomial of an arbitrary single zigzag chain $N(n)$, as illustrated in Figure 11 for $n=5$.

The highly regular structure of the interface connectivity graph for single zigzag chains allows us to obtain a general formula for $\operatorname{ZZ}(N(n), x)$ based on the solution of Equation (9). The set of recurrence relations given by Equation (9) can be rewritten in a more explicit form as

$$
\begin{aligned}
& W\left((\mathrm{ds})_{j+1}\right)=W\left((\mathrm{ds})_{j}\right)+W\left((\mathrm{sd})_{j}\right)+W\left((\mathrm{aa})_{j}\right), \\
& W\left((\mathrm{sd})_{j+1}\right)=W\left((\mathrm{ds})_{j}\right), \\
& W\left((\mathrm{aa})_{j+1}\right)=x \cdot W\left((\mathrm{ds})_{j}\right),
\end{aligned}
$$

which is valid also for $(\mathrm{ds})_{n+1} \equiv$ end, and can be folded to

$$
W\left((\mathrm{ds})_{j+1}\right)=W\left((\mathrm{ds})_{j}\right)+(1+x) \cdot W\left((\mathrm{ds})_{j-1}\right)
$$

with the initialization $W\left((\mathrm{ds})_{0}\right) \equiv W($ start $)=1$ and $W\left((\mathrm{ds})_{1}\right)=1$. Since for $n \in \mathbb{N}, W\left((\mathrm{ds})_{n+1}\right)=W($ end $)=$ $\mathrm{ZZ}(N(n), x)$, Equation (12) directly translates to the recurrence relation between $\mathrm{ZZ}$ polynomials

$$
\begin{aligned}
\mathrm{ZZ}(N(n), x)= & \operatorname{ZZ}(N(n-1), x) \\
& +(1+x) \cdot \operatorname{ZZ}(N(n-2), x)
\end{aligned}
$$

derived earlier for these structures by Zhang and Zhang (Equation (4.9) of Ref. [1]) and Chou and Witek (Equation (10) of Ref. [3], Equation (1) of Ref. [8], and Equation (20) of Ref. [4]). The solution of Equation (13) is given ${ }^{[3,4]}$ by

$$
\operatorname{ZZ}(N(n), x)=\sum_{k=0}^{n}\left(\begin{array}{c}
n+1-k \\
k
\end{array}\right)(1+x)^{k} .
$$

Alternative forms of this solution are given by Equation
(4.12) of Ref. [1], Equation (2) of Ref. [8], and Equation (11) of Ref. [3]. It is easy to verify that the explicit formula for the number of Clar covers of order $k$ in $N(n)$ is given by

$$
c_{k}=\sum_{j=0}^{\left\lceil\frac{n}{2}\right\rceil}\left(\begin{array}{c}
n+1-k-j \\
k+j
\end{array}\right)\left(\begin{array}{c}
k+j \\
k
\end{array}\right),
$$

which yields yet another alternative formulation of the $\mathrm{ZZ}$ polynomial of single zigzag chains given by

$$
\operatorname{ZZ}(N(n), x)=\sum_{k=0}^{\left\lceil\frac{n}{2}\right\rceil}\left(\sum_{j=0}^{\left\lceil\frac{n}{2}\right\rceil}\left(\begin{array}{c}
n+1-k-j \\
k+j
\end{array}\right)\left(\begin{array}{c}
k+j \\
k
\end{array}\right)\right) x^{k} .
$$

As can be seen from the derivations presented above, the interface connectivity graph methodology provides two independent techniques of computing the $\mathrm{ZZ}$ polynomials of single zigzag chains $N(n)$. The first technique relies on recursive computation of the generalized polynomial weight for each vertex of the graph and recognizing that the generalized polynomial weight of the end vertex is equivalent to the $Z Z$ polynomial of the whole structure. The second technique uses the interface connectivity graph for deriving an appropriate recurrence relation, whose solution produces a general, closed-form expression for the ZZ polynomial valid for an arbitrary value of $n$, in contrast to the first technique which is only capable of producing the actual values of the $\mathrm{ZZ}$ polynomial of all $N(j)$ with $j \leq n$ for some pre-selected value of $n$. Both techniques have their merits. The first one seems to be particularly useful for benzenoid structures without internal symmetry, for which the recurrence relation do not exist. If such relations do exist, the second technique, however, is much more powerful and should be preferred over the first one. 


\section{CONCLUSION}

In this manuscript we present a graph-theoretical interpretation of the recently developed interface theory of single zigzag chains $N(n)$. Based on the resulting graphs, we propose a novel, robust way of computing ZZ polynomials of $N(n)$. The interface theory allows one to characterize the global structure of any Clar cover of $N(n)$ using a sequence of local structure descriptors (fragments or interfaces). These local descriptors can be used to define certain directed graphs (connectivity graphs), such that the full set of walks on these graphs is isomorphic to the collection of Clar covers of $N(n)$. The set of vertices in the interface connectivity graph consists of interfaces together with two additional designated vertices, start and end. The set of directed edges reflects the connectivity properties between the interfaces as inferred from the interface theory. Connectivity graphs possess the remarkable property that the complete set of walks from a source denoted as start to a sink denoted as end is isomorphic to the complete set of Clar covers of $N(n)$. We demonstrate that the connectivity graphs can be constructed in a direct form, in which the number of vertices is growing linearly with the length $n$ of the single zigzag chain $N(n)$, and in a reduced form, in which the number of vertices is independent of the actual length of the chain. The reduced interface connectivity graph has a particularly compact structure and consists only of five vertices and nine oriented edges. The full set of walks of length $n$ on this graph represents the full set of Clar covers of $N(n)$. The interface connectivity graph also allows a straightforward construction of the full set of Kekulé structures of $N(n)$. As a result, from the connectivity pattern of the interface connectivity graph, it is possible to derive an alternative explanation of the fact that Fibonacci cubes are the resonance graphs of single zigzag chains.

From the interface connectivity graph of $N(n)$, the ZZ polynomial of $N(n)$ can be robustly computed. The number of Clar covers of order $k$ corresponds to the number of walks that pass through exactly $k$ vertices associated with the interface (aa). In order to extract the ZZ polynomial from the interface connectivity graph, each vertex is assigned a polynomial weight. These weights are related by a set of simple recurrence relations. As a result, the $\mathbf{Z Z}$ polynomial of $N(n)$ can be readily and robustly determined by recursive computation of the weights. The proposed method does not require constructing the full set of Clar covers. Instead, since the set of Clar covers is encoded in the properties of the interface connectivity graph, the computation of the $\mathrm{ZZ}$ polynomial can be performed in a very compact fashion. Owing to the highly regular structure of the interface connectivity graph for $N(n)$, it is possible to find a closed-form solution of the recurrence relation for the vertex polynomial weights, yielding the ZZ polynomial of $N(n)$ for an arbitrary length $n$. Generalization of the interface theory and the connectivity graphs, including different methods of computing $\mathrm{ZZ}$ polynomials, to arbitrary benzenoid structures will be presented in the near future.

\section{REFERENCES}

[1] H. P. Zhang, F. J. Zhang, Discr. Appl. Math. 1996, 69, 147-167.

[2] I. Gutman, B. Furtula, A. T. Balaban, Polycyclic Aromat. Compd. 2006, 26, 17-35.

[3] C. P. Chou, H. A. Witek, MATCH Commun. Math. Comput. Chem. 2012, 68, 3-30.

[4] C. P. Chou, H. A. Witek, MATCH Commun. Math. Comput. Chem. 2014, 71, 741-764.

[5] I. Gutman, S. Gojak, B. Furtula, S. Radenković, A. Vodopivec, Monatsh. Chem. 2006, 137, 1127-1138.

[6] S. Gojak, I. Gutman, S. Radenković, A. Vodopivec, J. Serb. Chem. Soc. 2007, 72, 673-679.

[7] D. D. Chen, H. Y. Deng, Q. Z. Guo, MATCH Commun. Math. Comput. Chem. 2010, 63, 401-410.

[8] C. P. Chou, Y. T. Li, H. A. Witek, MATCH Commun. Math. Comput. Chem. 2012, 68, 31-64.

[9] L. Q. Xu, F. J. Zhang, Z. Naturforsch. 2012, 67a, 550-558.

[10] H. P. Zhang, W. C. Shiu, P. K. Sun, MATCH Commun. Math. Comput. Chem. 2013, 70, 477-492.

[11] C. P. Chou, H. A. Witek, MATCH Commun. Math. Comput. Chem. 2014, 72, 75-104.

[12] M. Berlic, N. Tratnik, P. Žigert Pleteršek, MATCH Commun. Math. Comput. Chem. 2015, 73, 443-456.

[13] H. A. Witek, J. Langner, G. Moś, C. P. Chou, MATCH Commun. Math. Comput. Chem. 2017, 78, 487-504.

[14] Q. Z. Guo, H. Y. Deng, D. D. Chen, J. Math. Chem. 2009, 46, 347-362.

[15] C. P. Chou, H. A. Witek, J. Math. Chem. 2012, 50, 1031-1033.

[16] C. P. Chou, H. A. Witek, MATCH Commun. Math. Comput. Chem. 2014, 72, 105-124.

[17] C. P. Chou, J.-S. Kang, H. A. Witek, Discr. Appl. Math. 2016, 198, 101-108.

[18] C. P. Chou, H. A. Witek, MATCH Commun. Math. Comput. Chem. 2015, 73, 421-426.

[19] H. A. Witek, G. Moś, C. P. Chou, MATCH Commun. Math. Comput. Chem. 2015, 73, 427-442.

[20] I. Gutman, S. J. Cyvin, Introduction to the theory of benzenoid hydrocarbons, Springer, Berlin, 1989.

[21] E. Clar, The Aromatic Sextet, Wiley, London, 1972.

[22] F. J. Zhang, H. P. Zhang, Y. T. Liu, Chin. J. Chem. 1996, 14, 321-325. 
[23] H. P. Zhang, Discr. Math. 1997, 172, 163-173.

[24] H. P. Zhang, F. J. Zhang, Discr. Math. 2000, 212, 261269.

[25] I. Gutman, MATCH Commun. Math. Comput. Chem. 1985, 17, 75-90.
[26] S. J. Cyvin, I. Gutman, Kekulé structures in benzenoid hydrocarbons, Springer, Berlin, 1988.

[27] J. Langner, H. A. Witek, J. Math. Chem. 2018, Submitted.

[28] S. Klavžar, P. Žigert, Fibonacci Quart. 2005, 43, 269-276. 